\title{
Projeto de um Controlador PD Robusto para um Sistema Barra-Bola
}

\author{
Lindelmo Havallon da Silva Pinto Azevedo* \\ Marcia Lissandra Machado Prado** \\ * Departamento de Tecnologia, Universidade Estadual de Feira de \\ Santana,BA, (e-mail: havallon.ecomp@gmail.com). \\ ** Departamento de Tecnologia, Universidade Estadual de Feira de \\ Santana,BA, (e-mail: marcia.lissandra@gmail.com)
}

\begin{abstract}
This paper presents the design of a robust PD controller for a ball-beam system using modal interval analysis. The paper is divided into system modeling, robust PD controller design and analysis of the obtained results. The objective of this work is designing a controller that ensures the system meets the stability requirements, considering the uncertains in the parameters of the plant.

Resumo: Este artigo apresenta o projeto de um controlador PD robusto para um sistema barra-bola utilizando análise intervalar modal. O artigo está dividido em modelagem do sistema, projeto do controlador robusto PD e análise dos resultados obtidos. O objetivo desse trabalho é projetar um controlador que assegure que o sistema atenda aos requisitos de estabilidade, considerando as incertezas nos parâmetros da planta.
\end{abstract}

Keywords: Control Theory; Robust Control; Ball-Beam System; Interval Analysis; Modals Intervals.

Palavras-chaves: Teoria de Controle; Controle Robusto; Sistema Barra-Bola; Análise Intervalar; Intervalos Modais

\section{INTRODUÇÃO}

Os sistemas de controle têm sido essenciais na sociedade moderna. Suas aplicações podem ser encontradas facilmente à nossa volta, como por exemplo em navegações e controle de mísseis, aviões e até mesmo navios. Podem ser também encontrados nas indústrias em reguladores do nível de líquidos em reservatórios, temperaturas de caldeiras e em espessura do material fabricado Nise (2012).

O primeiro passo ao projetar um controlador é conhecer o modelo matemático do objeto de controle, com base nas leis da física. Geralmente o modelo pode ser não linear, o que o torna difícil de estudar. Por conta disso, muitas vezes aproximamos o modelo para uma equação linear. Entretanto, essas aproximações incorporam incertezas nos parâmetros da planta. A fim de garantir o funcionamento do controlador, em respeito às suas especificações, utilizam-se a teoria de controle robusto, descrevendo as incertezas em um intervalo fechado Ogata (2010); Prado and Paz (2008).

O sistema barra-bola é um sistema que consiste em manter em equilíbrio a bola sobre a barra em uma determinada posição. O sistema possui dois graus de liberdades. Um deles é a bola rodando, para direita e para esquerda, sobre a barra, e o outro é a barra girando, onde um ponto da barra é fixo em uma junta de rotação e o outro é fixo em um motor.

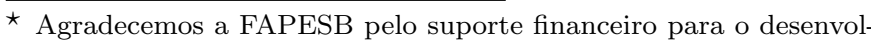
vimento deste trabalho.
}

Neste artigo é apresentado o projeto de um controlador proporcional e derivativo (PD) robusto para o sistema barra-bola utilizando a metodologia de análise intervalar modal descrita em Prado and Paz (2008). Para isso, foram aplicadas variações nos parâmetros do sistema, estabelecendo intervalos modais. Como os parâmetros do sistema estão descritos em intervalos é permitido que os mesmos se tornem variáveis. Assim, com o uso da aritmética intervalar modal é possível projetar o controlador robusto.

Este artigo está dividido da seguinte forma, na Seção 2 está representada uma breve fundamentação teórica necessária para o projeto, composta por: Controlador PD e análise intervalar modal. Na Seção 3 é apresentada toda a parte da modelagem do sistema barra-bola e o projeto do controlador PD robusto para o sistema. Na Seção 4 são apresentados os resultados obtidos neste projeto. E por fim, na Seção 5 estão descritas as conclusões provenientes deste trabalho.

\section{CONTROLE ROBUSTO PD UTILIZANDO ANÁLISE INTERVALAR MODAL}

\subsection{Controlador PD}

Considere o esquema de controle $\mathrm{PD}$ representado na Figura 1.

A parte proporcional representa um amplificador com um ganho $K_{p}$ ajustável e a parte derivativa introduz o elemento de predição no sinal de controle, assim permitindo melhorar o tempo de acomodação do sistema Ogata 


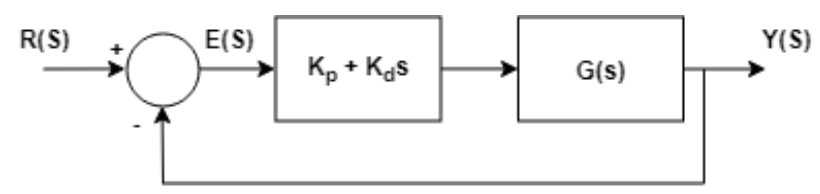

Figura 1. Diagrama de blocos do controlador PD.

(2010). A lei de controle no domínio do tempo é dada por Michael A. Johnson (2005):

$$
u_{c}(t)=k_{p} e(t)+k_{d} \frac{d e(t)}{d t}
$$

A função de transferência em malha fechada para este sistema é dada por:

$$
T(s)=\frac{G(s)\left(k_{p}+k_{d} s\right)}{1+G(s)\left(k_{p}+k_{d} s\right)}
$$

$\mathrm{O}$ controlador PD insere um zero no sistema em malha fechada. A adição desse zero pode aumentar a máxima sobre-elevação do sistema e com isso deixando-o mais oscilatório. Isso pode ser um problema para sistemas que não permitem uma alta sobre-elevação.

A solução deste problema ocasionado pelo controlador PD é a utilização da realimentação de velocidade representada pela Figura 2.

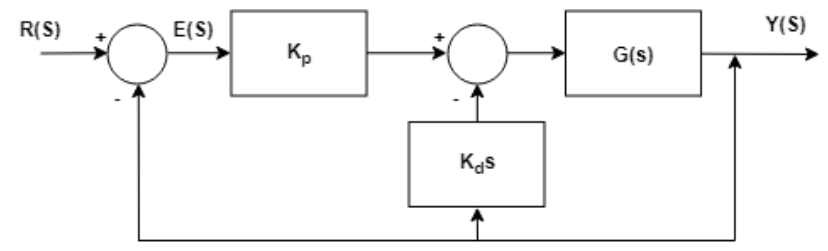

Figura 2. Diagrama de blocos do controlador PD com realimentação de velocidade.

A função de transferência em malha fechada para o sistema acima é dada por:

$$
T(s)=\frac{G(s) k_{p}}{1+G(s)\left(k_{p}+k_{d} s\right)}
$$

Assim não há acréscimo de zero no sistema e a sua resposta transitória tem um desempenho mais adequado. Com base nisso, foi utilizado este tipo de controlador neste trabalho.

\subsection{Análise Intervalar Modal}

Os intervalos modais são definidos por produtos cartesianos entre um conjunto dos intervalos fechados, $I(\mathbb{R})$, e um conjunto dos quantificadores existenciais e universais, $\{E, U\}$ Miguel A. Sainz (2014). Admitindo $[x]^{\prime} \in I(\mathbb{R})$ e $Q[x] \in\{E, U\}$, temos a representação dos intervalos modais:

$$
[x]=\left([x]^{\prime}, Q[x]\right)
$$

O intervalo fechado, $[x]^{\prime}$, representa a extensão de um intervalo modal e o quantificador, $Q[x]$, a modalidade que pode ser chamada de existencial, quando existe um $x \in[x]^{\prime}$, ou universal, para todo $x \in[x]^{\prime}$. As equações 5 e 6 representam os intervalos existenciais e universais, respectivamente.

$$
[x]=\left([x]^{\prime}, E\right)
$$

$$
[x]=\left([x]^{\prime}, U\right)
$$

Predicados são condições relacionadas a um intervalo, como por exemplo:

$$
y=x \in[x]^{\prime} \mid x \geq 0
$$

O predicado da sentença acima é a condição $x \geq 0$.

Para relacionar quantificadores aos predicados é utilizada uma notação especial:

$$
\begin{aligned}
& E\left(x,[a]^{\prime}\right) P(x) \\
& U\left(x,[a]^{\prime}\right) P(x)
\end{aligned}
$$

A equação 7 representa a relação do predicado com um quantificador existencial, enquanto a equação 8 representa a relação do predicado com um quantificador universal. Com isso, o conjunto dos intervalos modais é dado por:

$$
I^{*}(\mathbb{R})=\left\{[x]^{\prime},\{E, U\} \mid[x]^{\prime} \in I(\mathbb{R})\right\}
$$

Um intervalo modal pode ser classificado como intervalo próprio(10), impróprio(11) ou pontual(12).

$$
\begin{aligned}
& I^{*}(\mathbb{R})=\left\{[a, b] \in I^{*}(\mathbb{R}) \mid a \leq b\right\} \\
& I^{*}(\mathbb{R})=\left\{[a, b] \in I^{*}(\mathbb{R}) \mid a \geq b\right\} \\
& I^{*}(\mathbb{R})=\left\{[a, b] \in I^{*}(\mathbb{R}) \mid a=b\right\}
\end{aligned}
$$

As operações aritméticas intervalares modais têm semelhanças com às dos intervalos clássicos. Para um melhor detalhamento nas operações aritméticas e propriedades dos intervalos modais consulte Miguel A. Sainz (2014).

Considerando uma função $f$ real e continua em $\mathbb{R}^{k} \rightarrow \mathbb{R}$, sobre um intervalo $[a]$, qualquer função $F$ de $I^{*}\left(\mathbb{R}^{k}\right) \rightarrow$ $I^{*}(\mathbb{R})$ que satisfaça a propriedade da equação 13 , é chamada de extensão intervalar modal de $f$ Miguel A. Sainz (2014).

$$
\begin{array}{r}
U\left([x]^{\prime}, I^{*}\left(\mathbb{R}^{k}\right)\right)\left(\left(x \in[x]^{\prime}\right) \in P^{*}([a])\right) \Rightarrow \\
\left(f(x) \in f\left([x]^{\prime}\right)\right) \in P^{*}(F([a]))
\end{array}
$$

Adotando uma função $f$ continua em $\mathbb{R}^{k} \rightarrow \mathbb{R},\left(a_{p}, a_{i}\right)$ componentes de separação de $[a]=\left(\left[a_{p}\right],\left[a_{i}\right]\right) \mid[a] \in I^{*}(\mathbb{R})$, onde $\left[a_{p}\right]$ e $\left[a_{i}\right]$ são, respectivamente, sub-vetores próprios e impróprios de $[a]$, as extensões intervales semânticas modais $f^{*}$ e $f^{* *}$ são dadas pelas equações 14 e 15 , respectivamente Miguel A. Sainz (2014). 


$$
\begin{array}{r}
f^{*}([a])=\left[\min _{a_{p} \in\left[a_{p}\right]^{\prime}}\left\{\max _{a_{i} \in\left[a_{i}\right]^{\prime}} f\left(a_{p}, a_{i}\right)\right\},\right. \\
\left.\max _{a_{p} \in\left[a_{p}\right]^{\prime}}\left\{\min _{a_{i} \in\left[a_{i}\right]^{\prime}} f\left(a_{p}, a_{i}\right)\right\}\right] \\
f^{* *}([a])=\left[\max _{a_{p} \in\left[a_{p}\right]^{\prime}}\left\{\min _{a_{i} \in\left[a_{i}\right]^{\prime}} f\left(a_{p}, a_{i}\right)\right\},\right. \\
\left.\min _{a_{p} \in\left[a_{p}\right]^{\prime}}\left\{\max _{a_{i} \in\left[a_{i}\right]^{\prime}} f\left(a_{p}, a_{i}\right)\right\}\right]
\end{array}
$$

Sendo assim, se $f$ é unimodal para o intervalo $[a]$, ou seja $f$ é totalmente crescente ou decrescente em $[a]$, as extensões são iguais. Além disso, se $[a]$ é um intervalo próprio, logo $f^{*}$ e $f^{* *}$ são as imagens de $f$ no domínio $[a]^{\prime}$.

As extensões semânticas $f^{*}$ e $f^{* *}$ podem ser usadas para atribuir predicados aos resultados de cálculos intervalares. Para isso, é necessária a utilização dos teoremas semânticos.

Teorema 1. - Teorema semântico para $f^{*}$ Group (1998). Se $[a] \in I^{*}\left(\mathbb{R}^{k}\right), f$ é continua em $[a]^{\prime}$ e $F([a]) \in I^{*}(\mathbb{R})$, então $f^{*}([a]) \subseteq F([a])$ se somente se

$$
U\left(a_{p},\left[a_{p}\right]^{\prime}\right) Q(z, F([a])) E\left(a_{i},[a i]^{\prime}\right)\left(z=f\left(a_{p}, a_{i}\right)\right) .
$$

Teorema 2. - Teorema semântico para $f^{* *}$ Group (1998). Se $[a] \in I^{*}\left(\mathbb{R}^{k}\right), f$ é continua em $[a]^{\prime}$ e $F([a]) \in I^{*}(\mathbb{R})$, então $f^{* *}([a]) \supseteq F([a])$ se somente se

$$
U\left(a_{i},\left[a_{i}\right]^{\prime}\right) Q(z, \operatorname{Dual}(F([a]))) E\left(a_{p},\left[a_{p}\right]^{\prime}\right)\left(z=f\left(a_{p}, a_{i}\right)\right) .
$$

Geralmente o cálculo de $f^{*}$ e $f^{* *}$ é um trabalho difícil, o método usual é determinar aproximações externas de $f^{*}$ e internas de $f^{* *}$ de forma manter as interpretações semânticas dos Teoremas 1 e 2. Para isso, utilizam-se extensões racionais modais.

A extensão definida pela sequência de operações e indicada pela sintaxe de $f$ é chamada de extensão racional modal, $f_{R}([x])$, se $f$ for uma função contínua racional no domínio de $[x]^{\prime}$ Gardenes et al. (2001).

Há inúmeros resultados relacionando a extensão intervalar racional modal $f_{R}([x])$ às extensões semânticas $f^{*}$ e $f^{* *}$. Consulte Gardenes et al. (2001) para um melhor entendimento sobre como obter $f_{R}^{*}([x])$ e $f_{R}^{* *}([x])$, as extensões racionais modais equivalentes às aproximações externas e internas de $f^{*}$ e $f^{* *}$.

\section{MODELAGEM DO SISTEMA E PROJETO DO CONTROLADOR}

A metodologia de pesquisa adotada neste trabalho foi primeiramente realizar um estudo bibliográfico sobre teoria de controle, controle robusto, análise intervalar modal e sobre o sistema barra-bola. Logo em seguida, foi realizada a modelagem matemática do sistema barra-bola. Por fim, foi projetado o controlador robusto para a planta via análise intervalar modal e a obtenção de resultados através de simulações realizadas no MATLAB. As subseções a seguir descrevem com mais detalhes o processo de modelagem e concepção do controlador.

\subsection{Modelagem do Sistema Barra-Bola}

O sistema barra-bola é associado a problemas de controle reais, como, por exemplo, estabilizar horizontalmente um avião durante um fluxo de ar turbulento Wang (2007).

Neste sistema, a bola é posicionada sobre a barra, de forma que ela possa rolar livremente. Uma alavanca é utilizada para fazer o interfaceamento entre o servo motor e a barra. O motor gira um ângulo $\theta$, que consequentemente faz com que a barra gire $\alpha$. Com a variação do ângulo horizontalmente, a gravidade atua sobre a bola fazendo-a rolar CTMS (2005). A Figura 3 ilustra o sistema.

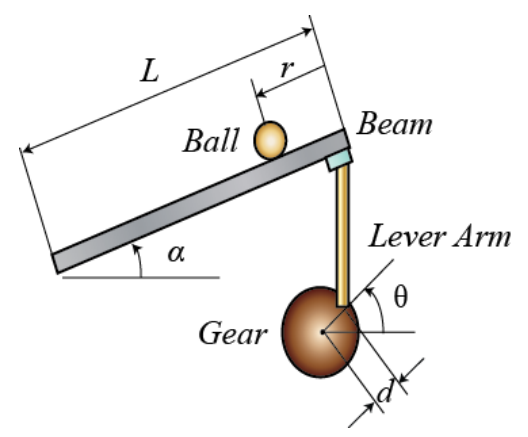

Figura 3. Sistema barra-bola CTMS (2005).

Utilizando a mecânica de Lagrange, a equação de movimento da bola é dada por Vincenty and Báez (2014):

$$
\left(\frac{J}{R^{2}}+m\right) \ddot{r}+m g \sin \alpha-m r \dot{\alpha}^{2}=0
$$

Onde $J$ é o momento de inércia da bola, $R$ o raio da bola, $m$ a massa da bola, $\ddot{r}$ a aceleração da bola, $g$ a aceleração gravitacional, $\dot{\alpha}$ a velocidade angular da barra, e $\alpha$ o ângulo horizontal da barra.

Utilizando a aproximação da função seno para ângulos pequenos, consulte Neto (2014) para mais detalhes, a aproximação linear do sistema é dada por:

$$
\left(\frac{J}{R^{2}}+m\right) \ddot{r}=-m g \alpha
$$

A relação entre o ângulo, $\alpha$, da barra e o ângulo, $\theta$, do motor é dada pela equação 20. Substituindo a equação 20 em 19 , temos a equação 21 que relaciona $\theta$ com a posição da bola $r$.

$$
\begin{gathered}
\alpha=\frac{d}{L} \theta \\
\left(\frac{J}{R^{2}}+m\right) \ddot{r}=-m g \frac{d}{L} \theta
\end{gathered}
$$

Sendo que $L$ é o comprimento da barra e $d$ o deslocamento da alavanca.

Aplicando a transformada de Laplace na equação 21 a fim de obter a função de transferência do sistema, temos: 


$$
G(s)=\frac{R(s)}{\Theta(s)}=-\frac{m g d}{L\left(\frac{J}{R^{2}}+m\right)} \frac{1}{s^{2}}
$$

Utilizando os valores das constantes do sistema que estão representados na Tabela 1, obtemos a seguinte equação, onde $K=0,21$ :

$$
G(s)=\frac{K}{s^{2}}
$$

Tabela 1. Valores dos parâmetros do sistema CTMS (2005).

\begin{tabular}{ccc} 
Simbolo & Valor & Unidade \\
\hline$m$ & 0,11 & $\mathrm{Kg}$ \\
$R$ & 0,015 & $\mathrm{~m}$ \\
$d$ & 0,03 & $\mathrm{~m}$ \\
$g$ & $-9,8$ & $\mathrm{~m} / \mathrm{s}^{2}$ \\
$L$ & 1 & $\mathrm{~m}$ \\
$J$ & $9,99 \times 10^{-6}$ & $\mathrm{~kg} \cdot \mathrm{m}^{2}$ \\
\hline
\end{tabular}

\subsection{Controlador PD Robusto}

A função de transferência do sistema utilizando o controlador PD com realimentação de velocidade é dada pela equação 3. Substituindo a planta pelo resultado encontrado na equação 23, temos:

$$
T(s)=\frac{K k_{p}}{s^{2}+K k_{d} s+K k_{p}}
$$

Como o maior expoente de $s$ no denominador é dois, podemos afirmar que o sistema é de segunda ordem. Assim, comparando o denominador da equação 24 com o polinômio característico, descrito na equação 25 , é possível obter os valores dos ganhos do controlador Ogata (2010). Logo, as equações 26 e 27 são as que definem o valor de $k_{d}$ e $k_{p}$ para este sistema.

$$
\begin{gathered}
s^{2}+2 \xi \omega_{n} s+\omega_{n}^{2} \\
k_{d}=\frac{2 \xi \omega_{n}}{K} \\
k_{p}=\frac{\omega_{n}^{2}}{K}
\end{gathered}
$$

Onde $\xi$ e $\omega_{n}$ são, respectivamente, o coeficiente de amortecimento e a frequência natural do sistema. Os valores destas constantes são determinados pelas seguintes equações Ogata (2010):

$$
\begin{gathered}
M P=e^{\frac{-\xi \pi}{\sqrt{1-\xi^{2}}}} \\
t_{s}=\frac{4}{\xi \omega_{n}}
\end{gathered}
$$

Sendo que o $M P$ é a máxima sobre-elevação do sistema e o $t_{s}$ é o tempo de estabelecimento necessário para que a resposta alcance e permaneça dentro da faixa de $2 \%$ do valor de regime. As especificações adotadas nesse projeto foram $M P \leq 5 \%$ e $t_{s} \leq 3 s$.
Para realizar o projeto de um controlador robusto para o sistema, é necessário transformar os parâmetros da planta em intervalos modais e garantir que para todos os valores dos parâmetros contidos nesses intervalos haja pelo menos um controlador que atenda aos requisitos do sistema. Sendo assim, foi considerada que a constante $K$, da planta(23) fosse variada em $5 \%$ para mais e para menos. $\mathrm{O}$ valor nominal $K=0,21$ foi transformado no intervalo $[K]^{\prime}=[0,1995,0,2205]$.

Para a obtenção dos intervalos para os requisitos do projeto, foram consideradas duas especificações dentro das adotadas anteriormente. A primeira é um $M P=0 \%$ e um $t_{s}=1,5 \mathrm{~s}$ e a segunda é um $M P=5 \%$ e um $t_{s}=3 \mathrm{~s}$. Sendo assim, os intervalos adotados foram $[\xi]^{\prime}=[1,0,6901] \mathrm{e}$ $\left[\omega_{n}\right]^{\prime}=[2,6667,1,9321]$.

Aplicando as propriedades e operações da aritmética intervalar modal encontradas em Miguel A. Sainz (2014) as equações que determinam os ganhos do controlador PD são dadas por:

$$
\begin{gathered}
{\left[k_{d}\right]^{\prime}=\left[\frac{2 \xi^{-} \omega_{n}^{-}}{K^{+}}, \frac{2 \xi^{+} \omega_{n}^{+}}{K^{-}}\right]} \\
{\left[k_{p}\right]^{\prime}=\left[\frac{\left(\omega_{n}^{2}\right)^{-}}{K^{+}}, \frac{\left(\omega_{n}^{2}\right)^{+}}{K^{-}}\right]}
\end{gathered}
$$

Sendo que $\xi^{-}, \omega_{n}^{-}$e $K^{-}$representam os extremos inferiores dos seus respectivos intervalos e $\xi^{+}, \omega_{n}^{+}$e $K^{+}$os extremos superiores.

Desenvolvendo as equações acima obtemos: $\left[k_{d}\right]^{\prime}=$ $[24,1875,13,3668]$ e $\left[k_{p}\right]^{\prime}=[32,2499,18,7112]$. É possível notar que os dois intervalos são impróprios. Logo, suas representações semânticas são:

$$
\begin{gathered}
U\left(K,[K]^{\prime}\right) E\left(\xi,[\xi]^{\prime}\right) E\left(\omega_{n},\left[\omega_{n}\right]^{\prime}\right) U\left(k_{d},\left[k_{d}\right]^{\prime}\right) k_{d}=\frac{2 \xi \omega_{n}}{K} \\
U\left(K,[K]^{\prime}\right) E\left(\omega_{n},\left[\omega_{n}\right]^{\prime}\right) U\left(k_{p},\left[k_{p}\right]^{\prime}\right) k_{p}=\frac{\omega_{n}^{2}}{K}
\end{gathered}
$$

Essas representações semânticas dizem que para todo $k_{d}$, existe pelo menos um valor de $\xi$ e $\omega_{n}$ para toda variação de $\mathrm{K}$ em seu intervalo. Do mesmo modo, para todo $k_{p}$ existe pelo menos um valor de $\omega_{n}$ para todo K. Ambas as semânticas satisfazem o predicado apresentado.

\section{RESULTADOS}

Tabela 2. Valores do parâmetro da planta dentro dos seus intervalos.

\begin{tabular}{cc} 
& $K$ \\
\hline Ponto mínimo & 0,1995 \\
Ponto central & 0,21 \\
Ponto máximo & 0,2205 \\
\hline
\end{tabular}

Para realizar a análise do controlador, foi utilizada a resposta ao degrau no sistema controlado, variando os parâmetros da planta. Os valores do parâmetro $K$, da planta foram variados de forma a representar os extremos 
do seu intervalo e o ponto central. Eles podem ser vistos na Tabela 2.

Os valores de $k_{d}$ e $k_{p}$ foram variados em um conjunto de cinco controladores dentro dos intervalos e aplicados a cada uma das variações da planta, tais valores podem ser vistos na Tabela 3.

Tabela 3. Valores dos ganhos do controlador dentro dos seus intervalos.

\begin{tabular}{ccc} 
& $k_{d}$ & $k_{p}$ \\
\hline Controlador 1 & 24,1875 & 32,2499 \\
Controlador 2 & 13,3668 & 18,7112 \\
Controlador 3 & 13,3668 & 25,4806 \\
Controlador 4 & 18,7771 & 32,2499 \\
Controlador 5 & 18,7771 & 25,4806 \\
\hline
\end{tabular}

A Figura 4 mostra o resultado obtido para o sistema barra-bola em que foram aplicados os valores de $[K]^{\prime}$, $\left[k_{d}\right]^{\prime}$ e $\left[k_{p}\right]^{\prime}$ na equação 24 . É possível notar, através do gráfico, que o erro de regime do sistema é nulo e que os controladores atenderam aos requisitos exigidos. Visto que a sobre-elevação e o tempo de acomodação respeitaram os limites adotados para este projeto.

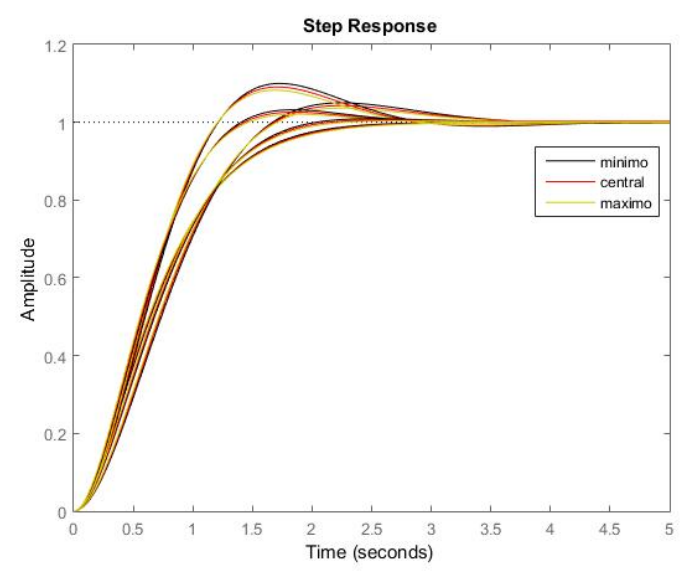

Figura 4. Resposta do sistema ao degrau utilizando o controlador PD robusto.

A Figura 5 mostra o resultado aplicando os cinco controladores na planta não-linear(18). Com a análise gráfica podemos perceber que com exceção do controlador 3 , que traz uma sobre-elevação de $10 \%$, os controladores provenientes dos intervalos $\left[k_{d}\right]^{\prime}$ e $\left[k_{p}\right]^{\prime}$ satisfazem o critério do sistema. Por conta de utilizar a planta nãolinear para aplicar os controladores, é esperado que alguns controladores possam não atingir o objetivo, sendo que os mesmos foram projetados com base do sistema linear. Entretanto, o resultado continua sendo excelente, visto que há a possibilidade de escolher diversos controladores a fim de encontrar um resultado mais adequado.

Também foram feitas análises com o controlador tradicional (24) com intuito de fazer comparações com o controlador robusto.

A Figura 6 mostra o resultado do controlador tradicional aplicado na planta com as variações de $K$ conforme a Tabela 2. Podemos notar que com o valor central de $K$ o controlador não consegue atender aos requisitos do

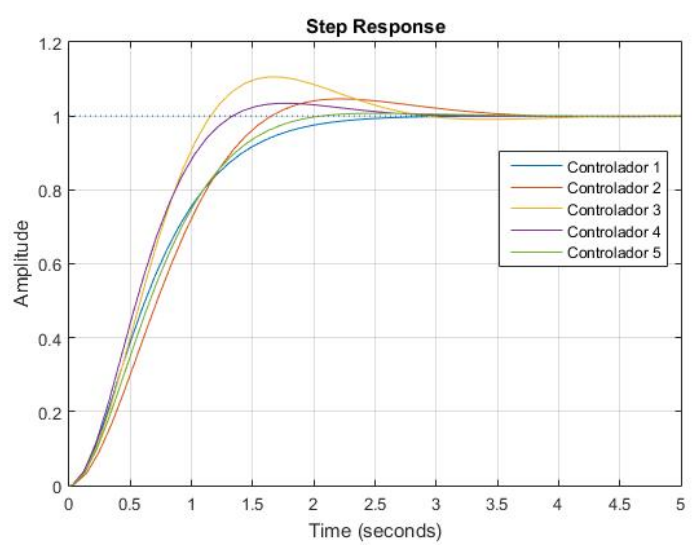

Figura 5. Resposta do sistema não-linear ao degrau utilizando o controlador PD Robusto.

sistema, atingindo uma sobre-elevação de $6 \%$ e tempo de acomodação de $3,1 s$.

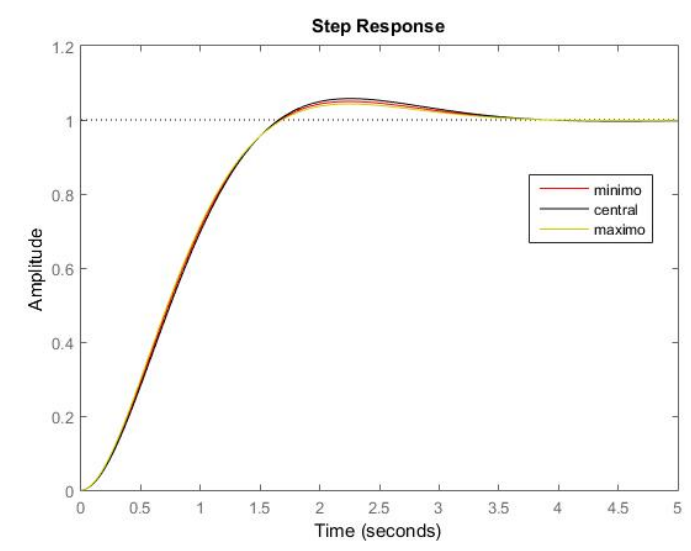

Figura 6. Resposta do sistema ao degrau utilizando o controlador PD tradicional.

A Figura 7 mostra o controlador tradicional atuando no sistema não-linear. Analisando o gráfico, é possível observar que o controlador tradicional não consegue atender às especificações do sistema, tendo uma sobre-elevação de $5,5 \%$ e um tempo de acomodação de 3,12s. Assim, o controlador robusto mostrou-se ser mais eficaz quando aplicado na situação em que os parâmetros da planta podem variar.

\section{CONCLUSÃO}

Com os resultados obtidos com os projetos dos controladores PD robusto e tradicional, foi possível perceber que em situações em que há variações na planta, o controlador robusto consegue garantir um desempenho melhor que o tradicional.

$\mathrm{O}$ projeto do controlador $\mathrm{PD}$ robusto utilizando análise intervalar modal mostrou-se bastante eficiente para ser aplicado em sistemas que possuem incertezas em seus parâmetros e exigem um desempenho adequado.

Como trabalho futuro já está em desenvolvimento a implementação da planta e outros projetos de controladores, como o controlador digital. Assim, além das simulações, 


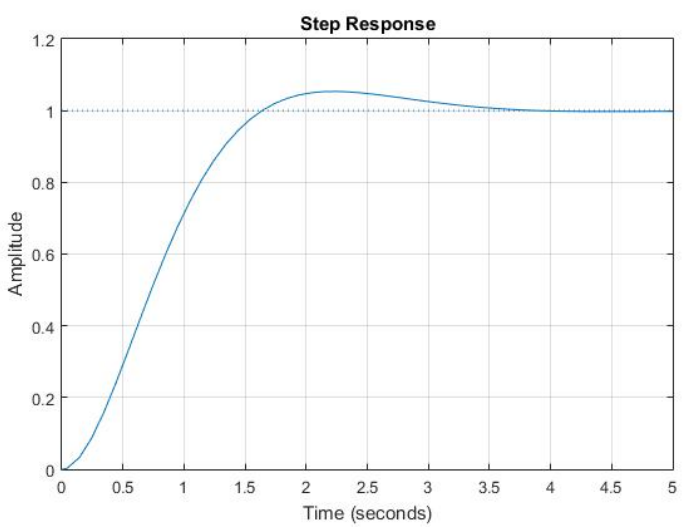

Figura 7. Resposta do sistema não-linear ao degrau utilizando o controlador tradicional.

poderemos aplicar os controladores na prática e analisar os seus resultados.

\section{AGRADECIMENTOS}

Agradecemos à UEFS por toda infraestrutura concedida.

\section{REFERÊNCIAS}

CTMS (2005). Ball \& beam: System modeling. Acessado em 04 abr. 2019. URL http://ctms.engin.umich. edu/CTMS/index $\cdot$ php? example=BallBeam\&section= SystemModeling.

Gardenes, E., Sainz, M., Jorba, L., Calm, R., Estela, R., Mielgo, H., and Trepat, A. (2001). Modal intervals. Reliable Computing 7: 77-111.

Group, S. (1998). Extensiones de las funciones continuas. Dept. de Informatica y Matematica Aplicada, Universidad de Girona, Spain.

Michael A. Johnson, M.H.M. (2005). PID Control: New Identification and Design Methods. Springer.

Miguel A. Sainz, Joaquim Armengol, R.C.P.H.L.J.J.V.a. (2014). Modal Interval Analysis: New Tools for Numerical Information. Lecture Notes in Mathematics 2091. Springer International Publishing, 1 edition.

Neto, E.C. (2014). Aplicação do polinômio de taylor na aproximação da função seno. Dissertação - Universidade Federal de Goiás Instituto de Matemática e Estatística.

Nise, N.S. (2012). Engenharia de Sistemas de Controle. LTC, 6 edition.

Ogata, K. (2010). Engenharia de Controle Moderno. Pearson Universidades, 5 edition.

Prado, M. and Paz, M. (2008). Controle robusto utilizando estrutura pd e análise intervalar modal. XIII Congresso Latinoamericano de Control Automatico.

Vincenty, C.G.B. and Báez, G.B. (2014). Modelling the ball-and-beam system from newtonian mechanics and from lagrange methods. Twelfth LACCEI Latin American and Caribbean Conference for Engineering and Technology.

Wang, W. (2007). Control of a ball and beam system. The University of Adelaide. 